\title{
Enhanced luminescence of organic/metal nanostructure for grating coupler active long-range surface plasmonic device
}

\author{
Nan-Fu Chiu and Chii-Wann Lin ${ }^{\text {a) }}$ \\ Department of Electrical Engineering, Institute of Biomedical Engineering, National Taiwan University, \\ No. 1, Sec. 4, Roosevelt Road, Taipei, 10617 Taiwan, Republic of China \\ Jiun-Haw Lee \\ Department of Electrical Engineering, Graduate Institute of Electro-Optical Engineering, National Taiwan \\ University, No. 1, Sec. 4, Roosevelt Road, Taipei, 10617 Taiwan, Republic of China \\ Chieh-Hsiung Kuan \\ Department of Electrical Engineering, Graduate Institute of Electronics Engineering, National Taiwan \\ University, No. 1, Sec. 4, Roosevelt Road, Taipei, 10617 Taiwan, Republic of China \\ Kuang-Chong Wu and Chih-Kung Lee \\ Institute of Applied Mechanics, National Taiwan University, No. 1, Sec. 4, Roosevelt Road, Taipei, \\ 10617 Taiwan, Republic of China
}

(Received 3 January 2007; accepted 31 July 2007; published online 23 August 2007)

\begin{abstract}
This letter is intended to demonstrate the effect of coupled active long-range surface plasmon polaritons (SPPs) on the plasmonics response of a lamellar grating nanostructure with organic material on the surface. The phenomenon of nano-optics gives rise to a selective spectral response and a local field enhancement. The authors' fabricated device consists of coupled organic/metal nanostructure with specific width and symmetric dielectric structure. The interaction between organic/metal interface SPPs can allow specific directional emission rather than isotropic emission. The authors present recent experimental results and discuss potential applications of such an active plasmonic biosensor with enhanced resonance energy emission due to interactions on the organic/ metal nanograting. (C) 2007 American Institute of Physics. [DOI: 10.1063/1.2773961]
\end{abstract}

Surface plasmon resonance (SPR) is a charge-density oscillation that can exist on the interface of two media with dielectric constants of opposite signs, ${ }^{1}$ for instance, a metal and a dielectric. This phenomenon was discovered by Wood ${ }^{2}$ in metal grating in the early 1900s. Other than the traditional Kretschmann ${ }^{3}$ and Otto ${ }^{4}$ configurations, several SPR devices were based on multilayer coupled SPR, ${ }^{5-7}$ long-range SPR, ${ }^{8}$ and coupled plasmon waveguide resonance ${ }^{9}$ at biosensing application. Recently, enhanced emission of organic layer on metallic thin film mediated by energy transfer of coupled surface plasmon polaritons ${ }^{10-15}$ (SPPs) has been extensively investigated. This technique of SP-coupled emission ${ }^{16-24}$ (SPCE) has performed on many structures but not on multilayer structure.

In this letter, we present and discuss the physics of enhancing efficiency of light emission from symmetric organic dielectric films on a lamellar grating nanostructure by using the coupled long-range SPP in four-layer symmetric dielectric structure. It can excite long-range SPP modes on the $\mathrm{Alq}_{3} / \mathrm{Au}$ interface and then couple to the Au/air interface for the emission of light. In our experiments, we prepared a onedimensional (1D) pattern of nanostructure by an electronbeam lithography system (ELS-7500EX, ELIONIX Co.). Both two-layer and four-layer devices were fabricated by thermally evaporating $\mathrm{Alq}_{3}(50 \mathrm{~nm}) / \mathrm{Au}(20 \mathrm{~nm})$ and $\mathrm{Alq}_{3}$ $(50 \mathrm{~nm}) / \mathrm{Au}(20 \mathrm{~nm}) / \mathrm{Alq}_{3}(50 \mathrm{~nm}) / \mathrm{Au}(20 \mathrm{~nm})$, on Si wa-

\footnotetext{
a) Author to whom correspondence should be addressed; FAX: 886-233665268; electronic mail: cwlinx@ntu.edu.tw
}

fer with the grating structure of $400 \mathrm{~nm}$ linewidth and $800 \mathrm{~nm}$ pitch width. Cross sections of the two-layer and four-layer devices are shown in Figs. 1(a) and 1(b), respectively. The $e$-beam resist thickness is $100 \mathrm{~nm}$ on silicon substrate with an exposure area of $1.2 \times 1.2 \mathrm{~mm}^{2}$. We have set up a reflectivity measurement system to measure the angular dependent photoluminescence (PL) of the surface plasmon coupled emission spectra produced by designated grating. All the experiments were performed at room temperature using the setup, as shown in Fig. 1(c).

Incident light with the wavelength of $405 \mathrm{~nm}$ from a white light source (Newport Oriel Spectral Luminator 69050) illuminates the sample through a polarization filter at fixed incident angle $\left(\theta_{i}\right)$ of $45^{\circ}$. The sample was kept at a fixed azimuthal angle $(\varphi)$ of $0^{\circ}$ incidence to pumping the organic molecules to excite metal grating and cross coupler produce SP-coupled emission. The emission beam is collected through a 2 in. lens with focal distance of $5 \mathrm{~cm}$ on a spectrometer (USB2000-Vis-NIR, Ocean Optics Co.) or a charge-coupled device camera (Hamamatsu C2400). The spectrometer can be programably controlled to measure the SP-coupled emission intensity at different emission angles $\left(\theta_{e}\right)$ in response to the optical signal change in SPPs as well as the surface refractive index changes on the SPPs-grating surface. This emission is scattered reradiation from $\mathrm{Alq}_{3}$-excited SPPs modes rather than direct radiation from $\mathrm{Alq}_{3}$ molecules.

The grating SPCE is based on light diffraction on a periodically modulated surface. The emission-coupling condition can be expressed by Eq. (1). 


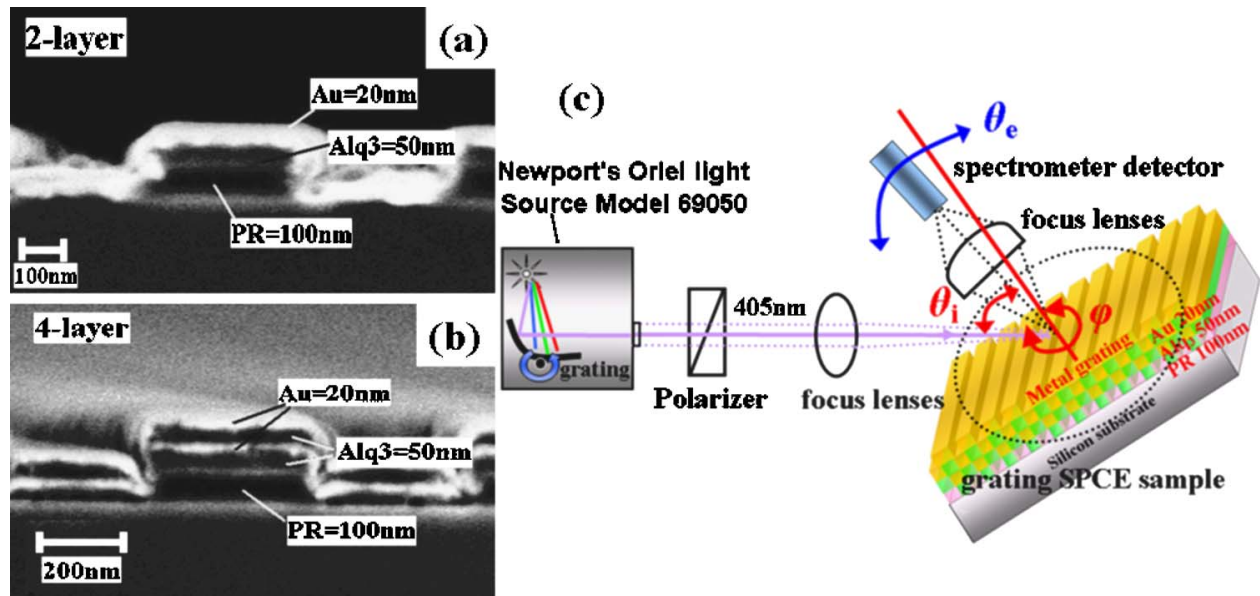

FIG. 1. (Color online) [(a) and (b)] Scanning electron microscopy images of the gratings cross section, which demonstrate the arrangement of a periodically lamellar layer. (a) is for twolayer structure and (b) is for four-layer structure. Schematic sketch shows the experimental setup for the PL SPcoupled emission from $\mathrm{Alq}_{3}$ through metal grating is shown as in (c).

$$
k_{\|}=k_{0} \sqrt{\varepsilon_{\mathrm{Alq}_{3}}} \sin \left(\theta_{e}\right)=k_{0} \sqrt{\frac{\varepsilon_{\mathrm{Au}} \cdot \varepsilon_{\mathrm{Air}}}{\varepsilon_{\mathrm{Au}}+\varepsilon_{\mathrm{Air}}}} \pm m \frac{2 \pi}{\Lambda}=k_{\mathrm{SPP}},
$$

where $k_{\|}$is the wave vectors parallel to the surface of the emission light, $k_{0}$ is the wave vector in vacuum, and $k_{\mathrm{SPP}}$ is $\mathrm{SP}$ wave vector parallel to the surface. $m$ is an integer and $\Lambda$ is the grating pitch. $\varepsilon_{\mathrm{Air}}$ and $\varepsilon_{\mathrm{Au}}$ are the dielectric constants of air and $\mathrm{Au}$, respectively. Equation (1) shows that the emission direction depends on the frequency.

It is also possible to directly couple the incident photons with wave-vector component $k_{\|}$at an azimuthal angle $(\varphi)$ to SPP with momentum conservation. It is also clear that $\theta$ will be different as $\varphi$ changes in order to satisfy the coupling condition. When $\varphi \neq 0$, by simple geometry, we find that the scalar equivalent of equation is ${ }^{20}$

$$
k_{\mathrm{SPP}}^{2}=n_{d}^{2} k_{0}^{2} \sin ^{2} \theta+\left(m \frac{2 \pi}{\Lambda}\right)^{2} \pm 2 n_{d} m \frac{2 \pi}{\Lambda} k_{0} \sin \theta \cos \varphi .
$$

The SP-coupled emission resulted from the oscillation of free charges on the metal surface and $\mathrm{Alq}_{3}$ emission electromagnetic wave is represented by Eq. (1). Due to the coupling of two SP modes between organic/metal and metal/air inter- faces, the SPs on $\mathrm{Alq}_{3} / \mathrm{Au}$ interface can be decoupled into air if the phase matching condition is satisfied. We have calculated the $\mathrm{Alq}_{3} / \mathrm{Au}$ and $\mathrm{Au} / \mathrm{Air} \mathrm{SPR}$ radiation spectrum for each of our samples by measuring the intensity of the grating components emitted at their corresponding angles as determined by Eqs. (1) and (2). The PL spectra and corresponding luminescence changes at different emission angles are measured and shown as in Figs. 2(a) and 2(b). The enhanced PL can be due to the SPR effect as mentioned above.

We also examined the effect of surface plasmon coupling on the angular dispersion of emission wavelength. The resonance peaks for the coupling grating were obtained from rotary table measuring azimuthal angle $\varphi=0^{\circ}$. The emission spectra were measured at different angles $\left(\theta=-15-10^{\circ}\right)$. The enhanced emission resonance spectra measured with the coupling of different angles are shown in Figs. 3(a) and 3(b). The average shift in peak wavelength is $14 \mathrm{~nm} / \mathrm{deg}$ for the grating with a pitch of $800 \mathrm{~nm}$. The wavelength may shift from 700 to $480 \mathrm{~nm}$. Emission intensity at different viewing angles of the planar, two-layer, and four-layer devices are shown in Fig. 3(c). We can see that the maximum intensity of planar:two-layer:four-layer devices is 1:4:6. The total emission from the four-layer structure can be strongly enhanced

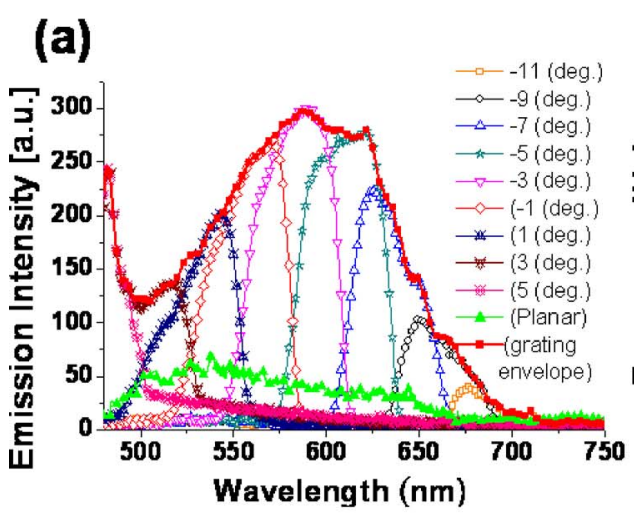

(b)

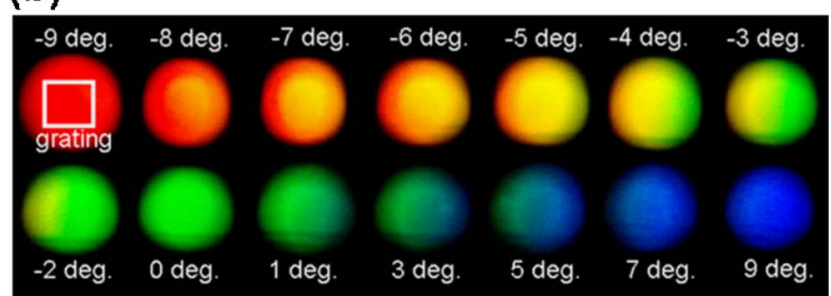

FIG. 2. (Color online) PL emission obtained from a grating four-layer sample, grating size (line of $400 \mathrm{~nm}$, pitch of $800 \mathrm{~nm}$, and area size of 1.2 $\times 1.2 \mathrm{~mm}^{2}$ ). (a) shows the PL of grating and nongrating (planar) samples as well as the integration of overall emission angles (envelope). The PL is measured on organic/metal grating. The changes in SPR were measured from the color of different angular spectra shown in (b). (c) shows the relation between frequency and wave vector. The fitting results are close to the theoretical dispersion relation. The data were taken from the sample with $800 \mathrm{~nm}$ grating pitch. 


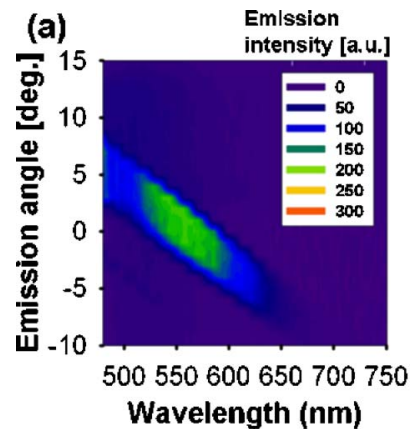

(c)



FIG. 3. (Color online) PL three-dimensional emission image obtained from a grating sample. The dependence of the emission spectra on observation angle $(\theta)$ is shown in (a) and (b) for two-layer and four-layer structures, respectively. (c) shows the experimental directional characteristic diagram of PL emission in polar coordinates of planar (circle), two-layer (triangle), and four-layer structures (square).

by recovering coupled SPP from $\mathrm{Alq}_{3}$ emission and scattering with the grating nanostructure.

With these data, we can use peak emission wavelength of each the resonant angles to fit the dispersion relation of Eq. (1). By fitting our data to this equation with $m=1, \Lambda$ $=800 \mathrm{~nm}$, and wavelength at $550 \mathrm{~nm}, \mathrm{Alq}_{3}(n=1.724266$, $k=0.00334)$, and $\mathrm{Au}(n=0.3557, k=2.6956)$, we found that the experimental results is close to the theoretical dispersion relation $\left(K_{\mathrm{SPP}}\right)$ of a SP-coupled emission at Au/air interface. The fitted results are shown in Fig. 2(c).

Figure 4 shows the full width at half maximum (FWHM) of the PL spectrum at different emission wavelength angles. For the four-layer structure, the FWHM ranges from 40 to $50 \mathrm{~nm}$. This value is narrower than that of two-layer

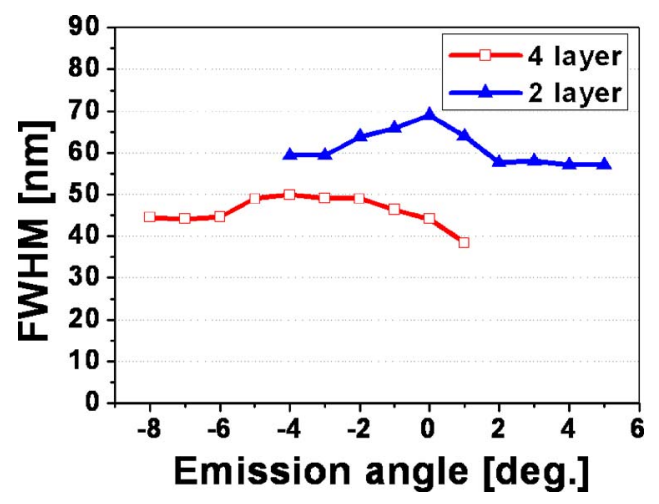

FIG. 4. (Color online) FWHM vs emission angles for two-layer and fourlayer devices. structure, i.e., $57-70 \mathrm{~nm}$, which may come from the stronger intensity of the four-layer devices.

We have demonstrated the phenomenon of symmetric dielectric layers for grating coupler active SP coupled emission of 1D rectangular lamellar grating in an organic/metal interface, which is employed to scatter SPPs modes to light. We have shown experimentally that strong coupling photonic resonance in SPPs grating resonances in $\mathrm{Alq}_{3} / \mathrm{Au}$ and $\mathrm{Au}$ /air symmetric mode leads to the formation of long-range SPP devices. The resonant intensity was enhanced up to six times on the four-layer device and the FWHM was less than $50 \mathrm{~nm}$. It shows possible applications by using long-range SPPs with the integration of electroluminescence optical devices for various applications, such as biosensors, organic light emitting diodes, and organic solar cells.

The authors would like to thank Hung-Chun Chang for his valuable suggestions and Shou-Yu Nien and Chun Yu, for their assistance in the measurements. This project is supported in part by National Science and Technology Program in Pharmaceuticals and Biotechnology, National Science Council, Taiwan, R.O.C., Under Grant Nos. NSC 95-2323B002-001, NSC 95-2323-B002-004, NSC 95-2221-E-002305, and MOEA 95-EC-17-A-05-S1-0017.

${ }^{1}$ H. Raether, Surface Plasmons on Smooth and Rough Surface and on Gratings (Springer, Berlin Heidelberg, 1988).

${ }^{2}$ R. W. Wood, Philos. Mag. 4, 396 (1902).

${ }^{3}$ E. Kretschmann, Z. Naturforsch. B 241, 313 (1971).

${ }^{4}$ A. Otto, Z. Phys. 216, 398 (1968).

${ }^{5}$ C.-W. Lin, K.-P. Chen, S.-M. Lin, and C.-K. Lee, Sens. Actuators B 113, 169 (2006).

${ }^{6}$ C.-W. Lin, K.-P. Chen, M.-C. Su, T.-C. Hsiao, S.-S. Lee, S.-M. Lin, X.-J. Shi, and C.-K. Lee, Sens. Actuators B 117, 219 (2006).

${ }^{7}$ C.-W. Lin, K.-P. Chen, M.-C. Su, C.-K. Lee, and C.-C. Yang, Opt. Quantum Electron. 37, 1423 (2005).

${ }^{8}$ J. W. Attridge and I. A. Shanks, U.S. Patent No. 5,478,755 (26 December 1995).

${ }^{9}$ Z. Salamon, H. A. Macleod, and G. Tollin, Biophys. J. 73, 2791 (1997).

${ }^{10}$ Z. Liu, N. Fang, T. Yen, and X. Zhang, Appl. Phys. Lett. 83, 5184 (2003).

${ }^{11}$ W. L. Barnes, J. Lightwave Technol. 17, 2170 (1999).

${ }^{12}$ D. K. Gifford and D. G. Hall, Appl. Phys. Lett. 80, 3679 (2002).

${ }^{13}$ J. Feng, T. Okamoto, and S. Kawata, Appl. Phys. Lett. 87, 241109 (2005).

${ }^{14}$ T. Nikolajsen, K. Leosson, I. Salakhutdinov, and S. I. Bozhevolnyi, Appl. Phys. Lett. 82, 668 (2003).

${ }^{15}$ J. Feng, T. Okamoto, J. Simonen, and S. Kawata, Appl. Phys. Lett. 90, 081106 (2007)

${ }^{16}$ J. R. Lakowicz, J. Malicka, I. Gryczynski, and Z. Gryczynski, Biochem. Biophys. Res. Commun. 307, 435 (2003).

${ }^{17}$ I. Gryczynski, J. Malicka, Z. Grycznski, and J. R. Lakowicz, Anal. Biochem. 324, 170 (2004).

${ }^{18}$ C. D. Geddes, I. Gryczynski, J. Malicka, Z. Gryczynski, and J. R. Lakowicz, J. Fluoresc. 14, 119 (2004).

${ }^{19}$ I. Pockrand, A. Brillante, and D. Möbius, Chem. Phys. Lett. 69, 499 (1980).

${ }^{20}$ S. C. Kitson, W. L. Barnes, and J. R. Sambles, Opt. Commun. 122, 147 (1996).

${ }^{21}$ J. Kalkman, C. Strohhofer, B. Gralak, and A. Polman, Appl. Phys. Lett. 83, 30 (2003).

${ }^{22}$ S. A. Maier, P. G. Kik, H. A. Atwater, S. Meltzer, E. Harel, B. E. Koel, and A. A. G. Requicha, Nat. Mater. 2, 229 (2003).

${ }^{23}$ G. Winter and W. L. Barnes, Appl. Phys. Lett. 88, 051109 (2006).

${ }^{24}$ P. Andrew and W. L. Barnes, Science 306, 1002 (2004). 